also higher among HIV patients, but it only presented a tendency to significance $(p=0.07)$. Regarding serum NGAL, no significant difference was observed between the groups $(\mathrm{p}=0.417)$. There was significant association between urinary NGAL andmicroalbuminuria $(r=0.378, p=0.003)$ and MCP-1 $(\mathrm{r}=0.476, \mathrm{p}<0.001)$ in all HIV patients.

Conclusion HIV patients usingcART presents subclinical kidney disease detected through novel biomarkers. KIM-1 may serve as early marker of tenofovir nephrotoxicity, and MCP-1 appears to be related with higher viral load.

\section{LB1.63 HIGH ACCEPTANCE OF CERVICAL SELF-COLLECTION FOR DETECTION OF HPV, HHV-2 AND HIV-1 IN WOMEN LIVING IN TAPAJÓS REGION, AMAZÔNIA, BRAZIL}

\begin{abstract}
1,3LLS Rodrigues, ${ }^{2}$ VS de Paula, ${ }^{3}$ MG Morgado, ${ }^{4}$ NS Oliveira, ${ }^{2}$ LRP Lima, ${ }^{5} \mathrm{~V}$ Sahasrabuddhe, ${ }^{6} \mathrm{AF}$ Nicol, ${ }^{3} \mathrm{JH}$ Pilotto. ${ }^{1}$ Health Collective Institute, Federal University of Western Pará, Santarém-Pará, Brazil; ${ }^{2}$ Molecular Virology Laboratory, Oswaldo Cruz Institute, Oswaldo Cruz Foundation, Rio de Janeiro, Brazil; ${ }^{3}$ AIDS and Molecular Immunology Laboratory, Oswaldo Cruz Institute, Oswaldo Cruz Foundation, Rio de Janeiro, Brazil; ${ }^{5} 4$ Post-graduation in Pathology, Fluminense Federal University; ${ }^{6}$ Division of Cancer Prevention, National Cancer Institute, National Institutes of Health (NIH/NCI/US), Laboratory Interdisciplinary of Medical Research, Oswaldo Cruz Institute, Oswaldo Cruz Foundation, Rio de Janeiro, Brazil
\end{abstract}

\subsection{6/sextrans-2017-053264.168}

Introduction Cervical self-collection is a safe and efficient method for detecting sexually transmitted infections (STIs). The study aims to verify the acceptance of cervical self-collection and the prevalence of HPV, HHV-2 and HIV-1 infection in women living in the Tapajós region, Amazônia, Brazil.

Methods Cross-sectional study with women attending in Santarém-Pará. The collection was performed between August 2015 and January 2017. Participants collected cervical scrapings and peripheral blood. Those who accepted, also performed cervical self-collection. Detection of HPV DNA was performed by nestedPCR with MY09/11 and GP5/6 $6^{+}$primers and typing was done by sequencing. Detection of $\mathrm{HHV}-2$ DNA was performed by real-time PCR with Taqman. Identification of anti-HIV-1/2 antibodies was made by Alere Determine Kit.

Results A total of 206 specimens were obtained from 112 women. The acceptance of cervical self-collection was $84 \%$ (94/112) and HPV DNA was identified in 39.4\% (37/94) of the samples. While the prevalence of HPV infection in cervical scraping was 32.1\% (36/112). All the women presented Papanicolaou negative for malignancy. The most prevalent types were HPV-16 and HPV-18. The overall prevalence of HHV-2 infection was $8.9 \%$. The concordance rate in the molecular diagnosis between cervical scraping and cervical self-collection was 65\% (26/40) for HPV and 50\% (4/8) for HHV-2. No woman had HIV-1 reactive serology.

Conclusion A high prevalence of HPV infection was found in women without dysplastic lesion. Cervical self-collection had high acceptance, moderate concordance rate in the detection of HPV DNA compared to cervical scraping, and alone it was more efficient in the detection of HPV. This is the first study in women living in Tapajós region and the findings strongly suggest that cervical self-collection may be a useful tool for increasing access to diagnosis of STIs and screening for cervical cancer in women living in the Amazon.

\section{LB1.64 CD64-MEDIATED PHAGOCYTOSIS OF HUMAN SYPHILITIC SERUM OPSONIZED TREPONEMA PALLIDUM BY HUMAN MACROPHAGES REQUIRED IFN-GAMMA}

${ }^{1,2} \mathrm{KL}$ Hawley, ${ }^{3} \mathrm{AR}$ Cruz, ${ }^{1,2,4} \mathrm{SL}$ Benjamin, ${ }^{2} \mathrm{CJ}$ La Vake ${ }^{5} \mathrm{M}$ LeDoyt, ${ }^{3} \mathrm{LG}$ Ramirez, ${ }^{1-}$ ${ }^{6} \mathrm{JD}$ Radolf ${ }^{1,4} \mathrm{JC}$ Salazar. ${ }^{1}$ Department of Paediatrics, UConn Health, Farmington, CT 06030 USA; ${ }^{2}$ Division of Infectious Disease and Immunology, Connecticut Children's Medical Centre, Hartford, CT 06106 USA; ${ }^{3}$ Centro Internacional de Entrenamiento e Investigaciones Médicas, Cali, Colombia; ${ }^{5} 4$ Departments of Immunology; ${ }^{6}$ Medicine, Genetics and Developmental Biology, UConn Health, Farmington, CT 06030 USA

\subsection{6/sextrans-2017-053264.169}

Introduction Syphilis is a multi-stage, sexually transmitted disease caused by the spirochete Treponema pallidum (Tp). Clinical manifestations result from the treponeme's ability to elicit a robust immune response while at the same time evading host defenses. Syphilitic lesions are comprised of a rich cellular infiltrate, which includes IFN-gamma (IFNg) producing $\mathrm{T}$ cells, NK cells and activated macrophages. We previously, we demonstrated that human syphilitic serum (HSS) promotes efficient uptake of $T p$ by human monocytes and that opsonophagocytosis of $T p$ markedly enhances cytokine production. The purpose of this study is to establish a potential role for macrophages and opsonic $\mathrm{Ab}$ in clearance of $T p$ and generation of tissue-based inflammation during human syphilis.

Methods We used monocyte-derived macrophages to develop an ex vivo model for studying spirochete-macrophage interactions. We used macrophage-colony stimulating factor and IFNg for macrophage maturation and evaluated the immunophenotypic modulations by flow cytometry. We assessed $T p$ uptake, in the presence or absence of HSS by confocal microscopy. We also determined the cellular responses initiated by opsonophagocytosis of $T p$ using targeted transcriptional array analysis and cytokine bead array.

Results IFNg polarisation of macrophages led to an increase in Fcg receptors (FcgRs) expression, phagocytosis of HSS opsonized $T p$ and cytokine production. Blockade of CD64 significantly diminished spirochetal uptake and pro-inflammatory cytokine secretion by the macrophages.

Conclusion Our ex vivo studies provide a potential role for macrophages in clearance of $T p$ during human syphilis. These data are the first to demonstrate that CD64 in the primary FcR involved in opsonophagocytosis of $T p$ and IFNg plays a critical role in the macrophages responsiveness following uptake of the spirochete. Moreover, our study results also provide an ex vivo surrogate system for use in future syphilis vaccine studies.

\section{LB1.65 FREQUENCY OF BROADLY NEUTRALISING ANTIBODIES IN HIV-1 CHRONICALLY INFECTED INDIVIDUALS IN UGANDAN CLADES A \& D}

Kintu Christopher. IAVI/UVRI, Uganda

\subsection{6/sextrans-2017-053264.170}

Introduction The study was aimed at assessing frequencies of neutralising antibodies in individuals affected with the commonest HIV-1 clades A and D in Uganda.

Methods This was a cross- sectional study of 83 HIV-1 chronically infected Anti- Retroviral Therapy (ART) naïve adults who were enrolled from Medical Research Council (MRC) cohort and The AIDS Support Organisation (TASO) Clinic in Entebbe. Samples of Plasma were tested for the 